\title{
ON THE ORDER SCALE OF A UNIFORM SPACE
}

\author{
D. C. KENT
}

(Received 15 October 1981)

Communicated by J. H. Rubinstein

\begin{abstract}
The order topology is compact and $T_{2}$ in both the scale and retracted scale of any uniform space $(S, \mathscr{Q})$. If $(S, \mathcal{Q})$ is $T_{2}$ and totally bounded, the Samuel compactification associated with $(S, \mathcal{Q})$ can be obtained by uniformly embedding $(S, \mathcal{Q})$ in its order retracted scale (that is, the retracted scale with its order topology). This implies that every compact $T_{2}$ space is both a closed subspace of a complete, infinitely distributive lattice in its order topology, and also a continuous, closed image of a closed subspace of a complete atomic Boolean algebra in its order topology.
\end{abstract}

1980 Mathematics subject classification (Amer. Math. Soc.): 54 E 15, 54 F 05.

\section{Introduction}

The scale $(P, \mathscr{V})$ and retracted scale $\left(P_{0}, \widetilde{V}_{0}\right)$ of a uniform space were introduced in 1967, and subsequently the uniform and topological properties of these spaces have been studied fairly extensively (see [2], [3], [6], [8], [9], [10], and [11]). The set $P$ is the lattice of "semifilters" (formerly called "prefilters") which are subsets of Q ; the uniformity $\mathcal{V}$ on $P$ is derived from $\mathcal{Q}$ in a natural way. The retracted scale is also a uniform lattice obtained via a "Hausdorffization" of $(P, \mathcal{V})$. Applications of the scale, both inside and outside the realm of topology, are discussed by its originator, D. Bushaw, in [3].

The order scale (order retracted scale) refers to the lattice $P\left(P_{0}\right)$ in either its order topology or the unique compatible uniformity. The reason no systematic study of the order scales was undertaken sooner is, perhaps, that it seemed unlikely that they would reflect in any meaningful way the properties of the original uniform space. On the contrary, it turns out that every $T_{2}$, totally

C Copyright Australian Mathematical Society 1983 
bounded uniform space is uniformly embedded in its order retracted scale. This theorem and its corollaries constitute the main results of the paper. Another conclusion which emerges from this study is that the order scales give every indication of being as useful as the orginal scales in various applications. Finally, our results add weight to the consensus that the retracted scale is more applicable than the scale.

\section{Order convergence}

In this section, we define the relevant lattice concepts, and summarize some known results about order convergence which will be needed later in this paper. Throughout this section, $L$ will represent a complete lattice.

If $x \in L, A \subseteq L$, and $\mathscr{F}$ is a filter on $L$, then let:

$$
x^{*}=\{y \in L: y \geqslant x\}, \quad A^{*}=\cap\left\{x^{*}: x \in A\right\}, \quad \mathscr{F}^{*}=\bigcup\left\{F^{*}: F \in \mathscr{F}\right\} .
$$

The symbols $x^{+}, A^{+}, \mathscr{F}^{+}$designate the corresponding sets of lower bounds. If $x \leqslant y$, then $[x, y]=x^{*} \cap y^{+}$denotes the closed interval spanned by $x$ and $y$. A filter $\mathscr{F}$ on $L$ order-converges to $x$ if $x=\sup \mathscr{F}^{+}=\inf \mathscr{F}^{*}$. The order topology on $L$ has for its closed sets those sets $A$ which contain all of their order-convergence limit points. Order convergence does not always coincide with convergence in the order topology; when they do coincide, order convergence is said to be topological, and the resulting order topology is $T_{3}$. We shall see later that order convergence in the scale and retracted scale of any uniform space is always topological.

An element $x$ of $L$ is compact if $A \subseteq L$ and $x \leqslant \sup A$ implies that there is a finite subset $B$ of $A$ such that $x \leqslant \sup B$. An element with the dual property is said to be cocompact. $L$ is compactly (cocompactly) generated if each element of $L$ is the supremum (infimum) of a set of compact (cocompact) elements; if $L$ is both compactly and cocompactly generated, then $L$ is said to be bicompactly generated.

An element $y$ in $L$ is said to be an unavoidable lower (upper) bound of an element $x$ if $y$ belongs to every ideal $I$ (dual ideal $D$ ) such that $\sup I=x$ (inf $D=$ $x$ ). The set of all unavoidable lower (upper) bounds of $x$ is a (dual) ideal denoted by $I(x)(D(x))$.

The first proposition follows from the results of [7].

Proposition 1.1 If $L$ is bicompactly generated, then $I(x)=\bigcup\left\{y^{+}: y\right.$ compact, $y \leqslant x\}$ and $D(x)=\cup\left\{y^{*}: y\right.$ cocompact, $\left.x \leqslant y\right\}$.

The next result follows from Proposition 2, [4]. 
Proposition 1.2 If order convergence in $L$ is topological, then $x=\sup I(x)=$ inf $D(x)$, for all $x \in L$, and the neighborhood filter at $x$ is generated by closed intervals of the form $[a, b]$, where $a \in I(x), b \in D(x)$.

A subset $A$ of $L$ with the inherited order is called a subcomplete lattice of $L$ if, for every nonempty subset $B$ of $A, \sup _{A} B=\sup _{L} B$ and $\inf _{A} B=\inf _{L} B$. The next proposition is proved in [7].

Proposition 1.3 (a) If $L$ is bicompactly generated, then order convergence in $L$ is topological, and if $x \in L$, then the neighborhood filter at $x$ has an open base consisting of sets of the form $[a, b]$, where $a$ is a compact lower bound of $x$ and $b$ is $a$ cocompact upper bound of $x$. The order topology is totally disconnected.

(b) If $L$ is a subcomplete lattice of an atomic Boolean algebra, then $L$ is bicompactly generated and the order topology is, in addition, compact.

Let $L_{0}$ be a subset of $L$ with the inherited order relation which satisfies the followng conditions:

(c $c_{1} L_{0}$ contains the least element of $L$.

(c $\left.c_{2}\right)$ If $A \subseteq L_{0}, A \neq \varnothing$, then $\sup _{L} A=\sup _{L_{0}} A$.

$\left(\mathrm{c}_{3}\right)$ For $x \in L$, let $x_{0}=\sup \left(x^{+} \cap L_{0}\right)$. If $A \subseteq L, A \neq \varnothing$, and $x=\sup A$, then $x_{0}=\sup \left\{y_{0}: y \in A\right\}$.

Assuming that $L_{0}$ has the properties described above, define $\psi: L \rightarrow L_{0}$ by $\psi(x)=x_{0}$, all $x \in L$, where $x_{0}=\sup \left(x^{+} \cap L_{0}\right)$. The final proposition in this section is proved in [4].

Proposition 1.4 Let $L$ be a subcomplete lattice of an atomic Boolean algebra, let $L_{0}$ and $\psi: L \rightarrow L_{0}$ be as described in the preceding paragraph.

(a) For each $x \in L_{0}, \psi^{-1}(x)=\left[x_{0}, x^{0}\right]$, where $x^{0}=\sup \left\{y \in L: x_{0}=y_{0}\right\}$.

(b) Order convergence in $L_{0}$ is topological, the order topology on $L_{0}$ is compact, and, at each point $x \in L_{0}$, the neigborhood filter is generated by sets of the form $\left[a_{0}, b_{0}\right] \cap L_{0}$, where $a$ is a compact element in $L, a \leqslant x$, and $b$ is cocompact in $L$, $x^{0} \leqslant b$.

(c) $\psi: L \rightarrow L_{0}$ is continuous relative to the respective order topologies.

\section{The order scale}

A semifilter $\alpha$ on a set $S$ is a nonempty collection of subsets of $S$ such that $A \in \alpha$ and $A \subseteq B$ implies $B \in \alpha$. Given a uniform space $(S, \mathcal{Q} L$ ), let $P$ be the set of all semifilters on $S \times S$ which are subsets of $Q$; $P$ is partially ordered by the 
dual of set inclusion ( $\alpha \leqslant \beta$ if and only if $\beta \subseteq \alpha$ ). $P$ is a complete lattice with least element $\mathcal{Q}$ (which we shall denote by 0 ) and greatest element $\{S \times S\}$ (denoted by 1 ); infima and suprema in $P$ are set unions and intersections, respectively, and from this it follows that $P$ is infinitely distributive. Indeed, $P$ is a subcomplete lattice of the power set of the power of $S \times S$, with the dual of its usual ordering, which means that $P$ is a subcomplete lattice of an atomic Boolean algebra, and thus bicompactly generated.

For the remainder of this paper, $(S, Q)$ will represent an arbitrary uniform space unless otherwise indicated. With each $U \in \mathcal{Q}$, we associate two prefilters $\sigma_{U}$ and $\rho_{U}$ as follows:

$$
\begin{aligned}
& \rho_{U}=\{V \in \mathscr{U}: V \nsubseteq U\}, \\
& \sigma_{U}=\left\{V \in \mho_{U}: U \subseteq V\right\} .
\end{aligned}
$$

Proposition 2.1 (a) $\alpha \in P$ is compact if and only if there are entourages $U_{1}, \ldots, U_{n}$ in Q such that $\alpha=\rho_{U_{1}} \vee \cdots \vee \rho_{U_{n}}$.

(b) $\alpha \in P$ is cocompact if and only if there are entourages $U_{1}, \ldots, U_{n}$ in $Q$ such that $\alpha=\sigma_{U_{1}} \wedge \cdots \wedge \sigma_{U_{n}}$.

Proof. (a) If $\rho_{U} \leqslant \sup A$ for $A \subseteq P$, then some $\alpha \in A$ must exclude $U$, and so $\rho_{U}$ is compact. It is easy to check that the supremum of a finite set of compact elements is always compact. To see that all compact elements have the specified form, let $\beta \in P$ and observe that $\beta=\sup \left\{\rho_{U}: U \in \mathcal{U}\right\}, U \notin \beta$. If $\beta$ is compact, then $\beta$ must be the supremum of a finite number of the $\rho_{U}$ 's, which completes the proof of (a). The proof of (b) is similar.

By Proposition 1.3, order convergence in $P$ is topological; we denote the order topology by $\theta$. Properties of $(P, \theta)$ which follow from Proposition 1.3 and 2.1 are summarized in the next theorem.

THEOREM 2.2 For any uniform space $(S, \mathcal{Q}),(P, \theta)$ is a compact, $T_{2}$, totally disconnected topological space. If $\alpha \neq 0$, the $\theta$-neighborhood filter $\mathscr{U}_{\theta}(\alpha)$ at $\alpha$ has an open subbase of sets of the form $\left[\rho_{U}, \sigma_{V}\right]$, where $U \notin \alpha$ and $V \in \alpha . \mathcal{V}_{\theta}(0)$ has an open subbase of the form $\left[0, \sigma_{V}\right], V \in \mathcal{Q}$.

Since $(P, \theta)$ is compact and $T_{2}$, there is a unique uniformity $\theta$ for $P$ which induces $\theta$. We shall call $\theta$ the order scale topology and $\theta$ the order scale uniformity; the term order scale will be used ambiguously to mean either $(P, \theta)$ or $(P, \theta)$.

THEOREM 2.3 For any $(S$, U),$(P, \theta)$ is a uniform lattice. 
Proof. Since $\theta$ is the filter of neighborhoods of the diagonal in $(P, \theta) \times(P, \theta)$, it is sufficient to check that the lattice operations are continuous relative to order convergence in any infinitely distributive lattice. Since order convergence coincides with $\theta$, the result follows.

The description of $\mathfrak{T}_{\theta}(\alpha)$ in Theorem 2.2 might lead one to conjecture that the order scale is at least first countable whenever $(S, \mathcal{Q})$ is metrizable. This is incorrect, however, as we shall show after first restating a useful lemma proved in [11].

LEMMA 2.4 If $(S, Q)$ is neither finite nor indiscrete, $\boldsymbol{N}$ is the cardinality of $S$, and $a \in S$, then there is $V \in$ QU such that the cardinality of $S-V(a)$ is $\aleph$.

THEOREM $2.5(P, \theta)$ is first countable (second countable, metrizable) in its order topology if and only if $(S, \mathcal{Q})$ is either finite or indiscrete.

Proof. If $(S, \mathscr{Q})$ is finite or indiscrete, then $(P, \theta)$ is finite and discrete, and so has the three indicated properties. If $(S, \mathscr{Q})$ is neither finite nor indiscrete, then let $a \in S$ and $V \in \mathcal{Q}$ be as in Lemma 2.4. Let $\mathscr{B}$ be the set of all subsets of $(S-V(a)) \times\{a\}$, and for each $B \in \mathscr{B}$, let $V_{B}=V \cup B$. Let $\alpha=\inf \left\{\sigma_{\mathfrak{v}_{B}}: B \in\right.$ $\Re\}$. By a straightforward argument, one can verify $\Upsilon_{\theta}(\alpha)$ has no countable filter base. Consequently, $(P, \theta)$ is not first countable, second countable, or metrizable.

We next show that a uniformly continuous function between two uniform spaces can be "lifted" to a uniformly continuous function between their order scales. If $f:(S, \mathscr{Q}) \rightarrow\left(S^{\prime}, \mathscr{Q}^{\prime}\right)$ is uniformly continuous, then define $\hat{f}: P \rightarrow P^{\prime}$ (where $\left(P^{\prime}, \theta^{\prime}\right)$ denotes the order scale of $\left(S^{\prime}, \mathscr{Q}^{\prime}\right)$ ) as follows: if $\alpha \in P, \hat{f}(\alpha)=$ $[f(\alpha)] \cap \mathcal{Q}^{\prime}$, where $[f(\alpha)]$ is the smallest semifilter on $S^{\prime} \times S^{\prime}$ containing $\{f(A)$ : $A \in \boldsymbol{\alpha}\}$.

THeOREM 2.6 If $f:(S, \mathcal{Q}) \rightarrow\left(S^{\prime}, \mathcal{Q}^{\prime}\right)$ is uniformly continuous, then $\hat{f}:(P, \mathcal{Q}) \rightarrow$ $\left(P^{\prime}, \mathcal{O}^{\prime}\right)$ is also uniformly continuous.

Proof. It is sufficient to show that $\hat{f}:(P, \theta) \rightarrow\left(P^{\prime}, \theta^{\prime}\right)$ is continuous. Let $\alpha \in P$, and let $\left[\rho_{W}, \sigma_{V}\right]$ be a subbasic $\theta^{\prime}$-open neighborhood of $f(\alpha)$, where $W \notin \hat{f}(\alpha)$ and $V \in \hat{f}(\alpha)$. Let $T=f^{-1}(W)$ and $U=f^{-1}(V)$. One can easily verify that $\left[\rho_{T}, \sigma_{U}\right]$ is a neighborhood of $\alpha$ which maps under $\hat{f}$ into $\left[\rho_{W}, \sigma_{V}\right]$, establishing the continuity of $\hat{f}$ at $\alpha$. 


\section{The scale uniform space}

In this section, we review some basic properties of the scale uniform space $(P, \mathscr{V})$ introduced by Bushaw in [2], and make comparisons with the order scale $(P, \mathcal{Q})$.

Starting with an arbitrary uniform space $(S$, Q $)$, one associates with each entourage $U \in \mathcal{Q}$ the set $U^{\prime}=\{(\alpha, \beta) \in P \times P: A \in \alpha$ implies $U A \in \beta$ and $B \in \beta$ implies $U B \in \alpha\}$; the collection $\left\{U^{\prime}: U \in \mathcal{Q}\right\}$ is the base for a uniformity on $P$ which is called the scale uniformity $\widetilde{T}$. The scale topology $\tau$ is the completely regular topology on $P$ induced by $\mathcal{~}$. The term "scale of $(S, \mathcal{Q})$ " will be used ambiguously to mean $P,(P, \tau),(P, \mathscr{V})$; it should be clear from the context which is intended.

Like the order scale $(P, \mathcal{O})$, the scale $(P, \mathscr{V})$ is a uniform lattice which is complete both as a lattice and as a uniform space (see [6]). Unlike the order scale, $(P, \mathcal{V})$ is rarely $T_{2}$, compact, or totally disconnected. For $(P, \mathcal{V})$, the properties $T_{2}$, discrete, and totally disconnected are equivalent to each other and to the condition that $(S, \mathscr{Q})$ is discrete [8]; also, $(P, \mathscr{V})$ is compact if and only if $(S, \mathcal{Q})$ is either finite or indiscrete (see [6], [11]). The relationship between the scale and order scale uniformities is summarized in the next proposition, which is an easy consequence of preceding remarks and Theorem 2.2 .

Proposition 3.1 (a) $\mathcal{O} \leqslant \widetilde{V}$ if and only if $(S, \mathcal{Q}$ ) is discrete.

(b) $\mathcal{Q} \leqslant \mathcal{\Theta}$ if and only if ( $S, \mathcal{Q})$ is finite or indiscrete.

It is useful to compare the neighborhood filters $\mathfrak{V}_{\tau}(\alpha)$ for the scale topology with those described in Theorem 2.2 for the order scale topology. It is shown in [8] that $\Upsilon_{T}(\alpha)$ is generated by the "closed" lattice intervals of the form $\left[\alpha_{U}, \alpha^{U}\right]$, $U \in \mathcal{U}$, where $\alpha_{U}=\{V \in \mathcal{Q}: U V \in \alpha\}, \alpha^{U}=\{V \in \mathcal{U}: U A \subseteq V$ for some $A \in$ $\alpha\}$. Thus both $\mathcal{T}_{\theta}(\alpha)$ and $\mathcal{T}_{\tau}(\alpha)$ have filter subbases of "closed" lattice intervals; it should be noted, however, that the intervals $\left[\rho_{U}, \sigma_{V}\right]$ which generate $\mathcal{V}_{\theta}(\alpha)$ are both open and closed relative to $\theta$, while the intervals $\left[\alpha_{U}, \alpha^{U}\right]$ which generate $\tau_{\tau}(\alpha)$ are, in general, neither open nor closed relative to $\tau$.

Given $\alpha \in P$, define $\alpha_{0}=\sup \left\{\alpha_{U}: U \in \mathscr{Q}\right\}$ and $\alpha^{0}=\inf \left\{\alpha^{U}: U \in \mathcal{Q}\right\}$. The lattice interval $\langle\alpha\rangle=\left[\alpha_{0}, \alpha^{0}\right]$ is the equivalence class containing $\alpha$ relative to the equivalence relation: $\alpha \sim \beta$ if and only if $\alpha_{0}=\beta_{0} ; \mathscr{V}_{\tau}(\alpha)$ converges to each element of $\langle\alpha\rangle$ (see [6]). On the other hand, it is easy to check that the net $\left(\alpha_{U}\right)_{U \in \mathcal{Q}} \theta$-converges upward to $\alpha_{0}$, whereas the net $\left(\alpha^{U}\right)_{U \in \mathcal{Q}} \theta$-converges downward to $\alpha^{0}$. It follows that $\Upsilon_{T}(\alpha) \theta$-converges to $\alpha$ if and only if $\langle\alpha\rangle=\{\alpha\}$. Thus we have

Proposition 3.2 For any $\alpha \in P, \mathscr{T}_{\tau}(\alpha) \geqslant \mathscr{V}_{\theta}(\alpha)$ if and only if $\alpha_{0}=\alpha^{0}$ 
For any uniform space ( $S, \mathcal{Q} L$ ), there is at least one element $\alpha \in P$ such that $\alpha_{0}=\alpha^{0}$, namely $\alpha=0$. For this element, one can say more.

Proposition 3.3 For any uniform space $(S, \mathcal{Q}), \Upsilon_{\theta}(0)=\widetilde{V}_{\tau}(0)$.

Proof. By Theorem 2.2, $\mathcal{V}_{\theta}(0)$ has a filter subbase of the form $\left[0, \sigma_{V}\right], V \in \mathcal{Q}$. Since $0_{U}=0$ for all $U \in \mathcal{Q}, \Upsilon_{\tau}(0)$ has a filter subbase of the form $\left[0,0^{U}\right]$, $U \in \mathcal{Q}$. A simple computation shows that $V^{2} \subseteq U$ implies $0^{V} \subseteq \sigma_{U} \subseteq 0^{U}$, and the conclusion follows.

In at least two applications of the scale, the construction of generalized Liapunov functions [1] and generalized metrization ([2] and [9]), the behavior of the scale in the vicinity of 0 is of crucial importance. Proposition 3.3 suggests that. at least for these applications, the order scale may be as useful as the scale.

A related result is due to 0 . C. Ramsey, [9].

Proposition 3.4 For any uniform space $\mathscr{Q}$, the following statements are equivalent.

(a) $(S, \mathcal{Q})$ is metrizable.

(b) $\mathscr{V}_{\theta}(0)$ has a countable filter base.

(c) $\mathcal{V}_{\tau}(0)$ has a countable filter base.

(d) $(P, \tau)$ is first countable.

(e) $(P, \mathscr{V})$ is metrizable.

Richardson and Wolf, [11], showed that the scale (like the order scale) is second countable if and only if $(S, Q)$ is either finite or indiscrete.

\section{The order retracted scale}

The retracted scale uniform space is constructed in [6] via the standard "Hausdorffization" process in order to replace $(P, \mathcal{Y})$ by a uniform space which is $T_{2}$. In [6], the underlying set for the retracted scale is taken to be $P_{R}=\{\langle\alpha\rangle$ : $\alpha \in P\}$, where $\alpha=\left[\alpha_{0}, \alpha^{0}\right]$ is defined in Section 3. However Bushaw [2] chose to replace $P_{R}$ with the set $P_{0}=\left\{\alpha_{0}: \alpha \in P\right\}$ and to order $P_{0}$ by the order relation inherited from $P$. We shall use Bushaw's definition of the retracted scale, which is equivalent to the one used in [6].

Let $\psi: P \rightarrow P_{0}$ be defined by $\psi(\alpha)=\alpha_{0}$; then it turns out (see [2]) that $\psi(\mathcal{V})=\mathscr{V}_{0}$ is a uniformity on $P_{0}$ which coincides with the uniformity that $P_{0}$ inherits as a subspace of $(P, \mathscr{T})$. Thus $\left(P_{0}, \Upsilon_{0}\right)$ is a uniform retract of $(P, \mathcal{T})$. Let 
$\tau_{0}$ denote the associated retracted scale topology. By Proposition 6, [6], the operations sup and inf are preserved by $\psi$, and it follows easily that $P_{0}$ satisfies the conditions $\left(\mathrm{c}_{1}\right),\left(\mathrm{c}_{2}\right),\left(\mathrm{c}_{3}\right)$ of Section 1 relative to $P$. Let $\theta_{0}$ denote the order topology on $P_{0}$. By Proposition 1.4, $\psi:(P, \theta) \rightarrow\left(P_{0}, \theta_{0}\right)$ is continuous.

THEOREM 4.1 For any uniform space $(S, \mathscr{Q}),\left(P_{0}, \theta_{0}\right)$ is compact, $T_{2}$ and coincides with order convergence in $P_{0}$. At a point $\alpha \in P_{0}$, the neighborhood filter $\widetilde{\vartheta}_{\theta_{0}}(\alpha)$ has a filter subbase of intervals in $P_{0}$ of the form $\left[\left(\rho_{U}\right)_{0},\left(\sigma_{V}\right)_{0}\right]$, where $U \notin \alpha$ and $V \in \alpha^{0}$.

Proof. The first assertion follows directly from Proposition 1.4; it also follows from Lemma 1, [6] and Corollary 2.6, [5]. The second assertion follows from Proposition 1.4 and Theorem 2.2.

$\left(P_{0}, \theta_{0}\right)$ has a unique compatible uniformity which we denote by $\theta_{0}$; the term order retracted scale will be applied to either $\left(P_{0}, \theta_{0}\right)$ or $\left(P_{0}, \Theta_{0}\right)$.

TheOREM 4.2 For any uniform space $(S, \mathcal{Q}), \theta_{0} \leqslant \tau_{0}$ on $P_{0}$.

Proof. This follows immediately from Proposition 3.2 and the fact that $\psi$ preserves sups and infs.

In [6], it is shown that for an arbitrarily chosen $a \in S$, the function $v_{a}(x)$ : $(S, \mathscr{Q}) \rightarrow\left(P_{0}, \mathscr{V}_{0}\right)$, defined by $v_{a}(x)=\{U \in \mathcal{Q}:(a, x) \in U\}$, is a uniform embedding of a $T_{2}$ uniform space $(S, \mathscr{U})$ into its retracted scale. The next theorem and its corollaries are the main results of this paper.

THEOREM 4.3 If $(S, \mathscr{Q})$ is $T_{2}$ and totally bounded, then $v_{a}:(S, \mathscr{Q}) \rightarrow\left(P_{0}, \theta_{0}\right)$ is a uniform embedding.

Proof. Let $A$ be the $\tau_{0}$-closure of the range of $v_{a}$ in $P_{0}$. Since $\left(P_{0}, \tau_{0}\right)$ is complete by Theorem 2 [6] our assumptions about ( $S, \mathcal{Q}$ ) along with Lemma 4 [6] imply that $A$ is a compact $T_{2}$ subspace of $\left(P_{0}, \tau_{0}\right)$. The unique uniformity for $A$ relative to this inherited topology must be a subuniformity of $\mathcal{T}_{0}$. But, by Theorem 4.2, it must also be a subuniformity of $\mathcal{O}_{0}$.

COROLLARY 4.4 For a Tychonoff topological space S, the Samuel compactification of $S$ associated with the totally bounded uniformity थ can be obtained by embedding $(S$, Q $)$ in its order retracted scale. 
The scale is an infinitely distributive lattice because its lattice operations are set unions and set intersections; the retracted scale is infinitely distributive because $\psi$ preserves sup's and inf's.

COROLlary 4.5 Each compact $T_{2}$ topological space is a closed subspace of a complete, infinitely distributive lattice in its order topology.

Corollary 4.6 Let ( $S$, थ ) be a $T_{2}$, totally bounded uniform space whose induced topology is not totally disconnected. Then:

(a) $\psi:(P, \theta) \rightarrow\left(P_{0}, \theta_{0}\right)$ is not neither a retraction nor an open map.

(b) The lattice $P_{0}$ is not bicompactly generated.

(c) $P_{0}$ is not a subcomplete lattice of $P$.

Proof. The proofs of these three assertions all depend on the fact that $\left(P_{0}, \theta_{0}\right)$ cannot be totally disconnected because this property is hereditary. Since $(P, \theta)$ is totally disconnected by Theorem $2.2, \psi$ cannot be a retraction or an open map, otherwise total disconnectedness would extend to $\left(P_{0}, \theta_{0}\right)$. $P_{0}$ cannot be bicompactly generated; otherwise $\left(P_{0}, \theta_{0}\right)$ would be totally disconnected by Proposition 1.3. $P_{0}$ cannot be a subcomplete lattice of $P$; otherwise, $P_{0}$ would be compactly generated.

Since $(P, \theta)$ and $\left(P_{0}, \theta_{0}\right)$ are both compact, $T_{2}$ spaces, $\psi:(P, \theta) \rightarrow\left(P_{0}, \theta_{0}\right)$ is a closed map; and $\left(P_{0}, \theta_{0}\right)$ is a topological (and uniform) quotient space of $(P, \theta)$. Recalling that the scale is a subcomplete lattice of a complete atomic Boolean algebra, we obtain our final corollary to Theorem 4.3.

COROLLARY 4.8 Every compact $T_{2}$ topological space is the image under a closed, continuous map of a closed subspace of a complete atomic Boolean algebra in its order topology.

\section{References}

[1] G. Birkhoff, Lattice theory, (Amer. Math. Soc. Colloq. Publ. 25 (third edition) New York, 1967).

[2] D. Bushaw, 'A stability criterion for general systems', Math. Systems Theory 1 (1967), 79-88.

[3] D. Bushaw, 'The scale of a uniform space', Proc. of the International Symp. on Topology and its Applications, Hercegnovi, Yugoslavia, Aug. 25-31, 1968.

[4] H. Dobbertin, M. Erné and D. C. Kent, 'A note on order convergence in complete lattices, to appear.

[5] M. Erné and S. Weck, 'Order convergence in lattices', Rocky Mountain J. Math. 10 (1980). 805-818. 
[6] D. C. Kent, 'On the scale of a uniform space', Inventiones Math. 4 (1967), 159-164.

[7] D. C. Kent and C. R. Atherton, 'The order topology in a bicompactly generated lattice', $J$. Austral. Math. Soc. 8 (1968), 345-349.

[8] G. C. Leslie and D. C. Kent, 'Connectedness in the scale of a uniform space', J. Austral. Math. Soc. 13 (1972), 305-312.

[9] O. C. Ramsey, Jr., Some properties of the scale of a uniform space, (Ph. D. Dissertation, Washington State University, 1967).

[10] G. D. Richardson, 'Connectedness in the scale of uniform subspaces of $R$ ', J. Austral. Math. Soc. 18 (1974), 461-463.

[11] G. D. Richardson and E. M. Wolf, 'Topological properties of the scale of a uniform space', $J$. Austral. Math. Soc., to appear.

\section{Department of Mathematics \\ Washington State University \\ Pullman, Washington 99164 \\ U.S.A.}

